\title{
GM-CSF mediates pain and disease in a mouse model of osteoarthritis
}

Hot-on-the-heels of their demonstration that granulocyte-macrophage colonystimulating factor (GM-CSF) has a key role in the pain and inflammation of a mouse model of rheumatoid arthritis (RA; see further reading), Andrew Cook and colleagues now show that this mediator is also critical for pain and disease in experimental osteoarthritis (OA). As targeting GM-CSF in RA is currently progressing well in clinical development, the investigators hope that the strategy might be extended to OA in future.

"Inflammatory pain, including that associated with arthritis, represents a significant unmet medical need," explains Cook, adding that OA-of which pain is an important symptom-is the most common rheumatic disease. To determine the role of GM-CSF in OA, the investigators used the collagenaseinduced mouse model of the disease, in which intra-articular injection of collagenase degrades ligaments, inducing joint instability and 'OA-like pathology'. Arthritic disease was assessed histologically in wild-type and GM-CSFdeficient $\left(C s f 2^{-/-}\right)$mice; synovitis at 2 weeks, and cartilage damage at 6 weeks, was substantially reduced in the absence of GM-CSF.

\section{Pain...was absent when GM-CSF was blocked... 77}

"Next we assessed whether collagenaseinduced pain was GM-CSF-dependent, using mice treated prophylactically with a neutralising antibody to GM-CSF," says Cook. Pain, gauged by measuring weight distribution, was absent when GM-CSF was blocked. Furthermore, it was reversible when the antibody was used therapeutically. Importantly for OA treatment prospects, "neutralisation of GM-CSF not only abolished the existing pain within 3 days (the earliest time point measured following monoclonal antibody treatment), but also led to significantly reduced cartilage damage," adds Cook.

Despite these encouraging data, much remains unknown about the analgesic effects of GM-CSF, including "the nature of the GM-CSF-dependent nociceptive system."

"Our results suggest that it would be worth exploring the importance of GM-CSF for pain and disease in other OA models and perhaps clinically for this form of arthritis," concludes Cook.

\section{Emma Leah}

Original article Cook, A. D. et al. Granulocyte macrophagecolony stimulating factor is a key mediator in experimental osteoarthritis pain and disease development. Arthritis Res. Ther. doi:10.1186/ar4037

Further reading Onuora, S. Granulocyte-macrophage colony-stimulating factor required for inflammatory and arthritic pain. Nat. Rev. Rheumatol. 8, 499 (2012) 